\title{
Carcinoma papilifero da tireoide e suas variantes histológicas associados à tireoidite de Hashimoto
}

\author{
Thyroid papillary carcinoma and histologic variants linked to Hashimoto disease
}

Murilo Pedreira Neves Junior'; Marcos Pedro Guedes Camandaroba²; Marco Antônio Cardoso de Almeida ${ }^{3}$; Julia Souto Miranda ${ }^{4}$

\begin{abstract}
unitermos resumo
Tireoide

Introdução e objetivo: A associação entre o carcinoma papilífero da tireoide e suas variantes e a

Patologia

tireoidite de Hashimoto $(\mathrm{TH})$ é bastante questionada no meio científico, pois compartilham diversos aspectos morfológicos, imuno-histoquímicos e biomoleculares. Os tumores da tireoide representam mais

Carcinoma de $90 \%$ de todos os cânceres endócrinos e são caracterizados por alterações genéticas, entre as quais

Tireoidite envolvem RET (rearranjos) e BRAS, RAS, P53 (mutações). Já a TH é uma doença autoimune, caracterizada por falência tireoidiana secundária à destruição autoimune e que apresenta alterações de genes, entre eles RET/PTC, RAS e FAS. O objetivo deste trabalho é descrever a associação do carcinoma papilífero da tireoide com a TH, correlacionando-os com os dados demográficos e suas variantes histológicas. Método: Realizou-se um estudo de série de 466 casos de pacientes com diagnóstico anatomopatológico de carcinoma papilífero da tireoide de 2000 a dezembro de 2008. Foram feitas aplicações de formulários aos casos, visando coletar os dados demográficos e suas variantes. Resultados: $\mathrm{O}$ estudo apresentou uma coexistência de $30 \%$ de TH em pacientes com carcinoma papilífero da tireoide. No sexo feminino, houve maior número de casos no grupo com TH, valor de $p=0,046$. Conclusão: A série de casos apresentada mostrou frequência de $30 \%$ de TH nos casos de carcinoma papilífero da tireoide, sugerindo uma associação, não apenas casual, que levanta a possibilidade de uma relação de causa e efeito entre tireoidite e desenvolvimento do carcinoma.
\end{abstract}

abstract

Introduction and objective: The association between papillary thyroid carcinoma and its variants and Hashimoto's thyroiditis is widely questioned in the scientific area, as they both share several morphologic, immunohistochemical and biomolecular aspects. Thyroid tumors represent over $90 \%$ of all endocrine cancers and are characterized by genetic changes involving RET (rearrangements) and BRAS, RAS, P53 (mutations). Whereas Hashimoto's thyroiditis is an autoimmune disease characterized by auto-immune destruction followed by thyroid failure and it presents RET/PTC, RAS and FAS alterations. This study aims to describe the association of Hashimoto's thyroiditis with papillary carcinoma thyroid, correlating them with demographic data and histological variants. Methods: It was conducted a case series study of 466 patients diagnosed with thyroid papillary carcinoma from January 2000 to December 2008. Case forms were filled up with the aim to collect demographic data and histological variants. Results: The present study showed a coexistence of 30\% of Hashimoto's thyroiditis in patients with papillary thyroid carcinoma. Among female patients there was a higher number of Hashimoto's thyroiditis cases, $p=0.046$. Conclusion: The case series study showed a frequency of 30\% of Hashimoto's thyroiditis in cases with papillary thyroid carcinoma suggesting a noncoincidental association, which raises the possibility of a cause and effect relation between thyroiditis and carcinoma development. key words

Thyroid

Pathology

Carcinoma

Thyroiditis

Hashimoto

'Médico anatomopatologista; mestre em Medicina Interna.

${ }^{2}$ Acadêmico de Medicina pela Escola Bahiana de Medicina e Saúde Pública (EBMSP).

${ }^{3}$ Mestre em Medicina; professor assistente da Faculdade de Medicina da Universidade Federal da Bahia (UFBA); chefe do Serviço de Anatomia Patológica e Citopatologia do Hospital São Rafael.

${ }^{4}$ Médica anatomopatologista do Hospital São Rafael. 


\section{Introdução}

Os tumores da tireoide representam mais de $90 \%$ de todos os cânceres endócrinos, sendo o carcinoma papilífero a sua forma mais comum e de melhor prognóstico ${ }^{(20,24)}$.

São caracterizados por alterações genéticas diferentes, entre as quais envolvem RET e TRK (rearranjos), BRAS, RAS e P53 (mutações) ${ }^{(6,7,11,20)}$. A alteração da proteína RET é das mais importantes, pois pode ser encontrada no tecido da tireoide de pacientes com doença inflamatória crônica, como a tireoidite de Hashimoto

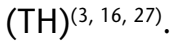

O carcinoma papilífero é definido como uma combinação de características morfológicas, entre elas o núcleo com contorno irregular, fendido, contendo inclusões, além de dispersão periférica de agregados de cromatina. Suas principais variantes histopatológicas são: variantes clássica, folicular, de células altas, de células colunares, sólida (esclerosante) e microcarcinoma papilífero $^{(1,5,15)}$.

Cada uma dessas variantes apresenta fenótipos diferenciados, com alterações nas taxas de morbidade e mortalidade ${ }^{(14)}$. As de maior agressividade incluem as variantes de células altas, sólida e de células colunares, sendo esta a mais grave, principalmente por invasão de linfonodos linfáticos, rápido crescimento, altas taxa de recorrência local e metástases para pulmão, cérebro e osso ${ }^{(12,14)}$.

A TH é uma doença autoimune, caracterizada por falência tireoidiana gradual secundária à destruição autoimune $^{(5,26)}$. Essas células tireoidianas não são individualmente normais e expressam alterações de genes, entre eles RET/PTC, RAS e FAS, implicados na alteração de função e morte celular ${ }^{(10,28)}$.

A glândula tireoide macroscopicamente se revela aumentada de tamanho, a cápsula está quase sempre intacta e por vezes nodular; microscopicamente há extensa infiltração linfocitária do parênquima com pequenos linfócitos, plasmócitos ${ }^{(5)}$.

A associação entre o carcinoma papilífero e suas variantes e a TH é bastante questionada no meio científico, pois compartilham diversos aspectos morfológicos, imuno-histoquímicos e biomoleculares ${ }^{(2,4)}$.

Os carcinomas papilíferos expressam frequentemente rearranjos genéticos durante a transformação maligna, exibindo expressão do RET/PTC e fusão de proteínas, sendo que também podem ser encontrados no tecido com a $\mathrm{TH}^{(1,6,9)}$. Esse fato foi observado inicialmente em pacientes expostos à radiação atômica do acidente de Chernoby|(26). A observação da presença de infiltração linfocitária na tireoide com carcinoma papilífero sugere que antígenos expressados por células do tumor sejam responsáveis por induzir uma resposta imune antitumoral ${ }^{(6,9,21)}$.

\section{Objetivo}

O objetivo deste trabalho é descrever a associação do carcinoma papilífero da tireoide com a TH, diagnosticada no serviço de Anatomia Patológica e Citopatologia do Hospital São Rafael, correlacionando-os com os dados demográficos e as suas variantes histológicas.

\section{Material e método}

Foi realizado um estudo de casos, aprovado no conselho de ética do Hospital São Rafael, de pacientes com carcinoma papilífero da tireoide, em material histopatológico diagnosticado no serviço de Anatomia Patológica e Citopatologia do Hospital São Rafael, de janeiro de 2000 a dezembro de 2008. Foram selecionados todos os casos com carcinoma papilífero da tireoide com e sem TH. Os critérios de exclusão foram casos com dados perdidos ou incompletos.

O estudo baseou-se na revisão dos prontuários médicos e na aplicação de um formulário aos casos de carcinoma papilífero da tireoide, visando coletar dados demográficos, como idade, sexo, data do diagnóstico, e suas variantes encontradas no anatomopatológico. As variantes histológicas do carcinoma papilífero consideradas no trabalho foram: variante clássica, variante folicular, variante de células altas, variante de células clara e variante sólida, bem como microcarcinoma. Consideramos microcarcinoma as lesões com menos de $1 \mathrm{~cm}$ no maior diâmetro.

Em uma segunda etapa, as lâminas dos exames anatomopatológicos foram revisadas pelos dois patologistas deste estudo, em microscópio Nikon Eclipse E-200.

Os resultados dos formulários aplicados aos casos foram analisados após confecção do banco de dados no software SPSS versão 9. Foi procedida análise descritiva de todas 
as variáveis com o estudo do qui-quadrado, tendo como comparação significativa o valor de $p<0,05$.

\section{Resultados}

No período de 2000 a 2008, foram realizadas 1.438 tireoidectomias no centro cirúrgico do Hospital São Rafael. Foram selecionados 466 casos com diagnóstico de carcinoma papilífero. Desses, 140 apresentavam coexistência de carcinoma papilífero da tireoide e TH (30\%), enquanto 346 casos não apresentavam tireoidite (70\%). (Figura). Foram excluídos seis casos que apresentavam doença metastática para tireoide.

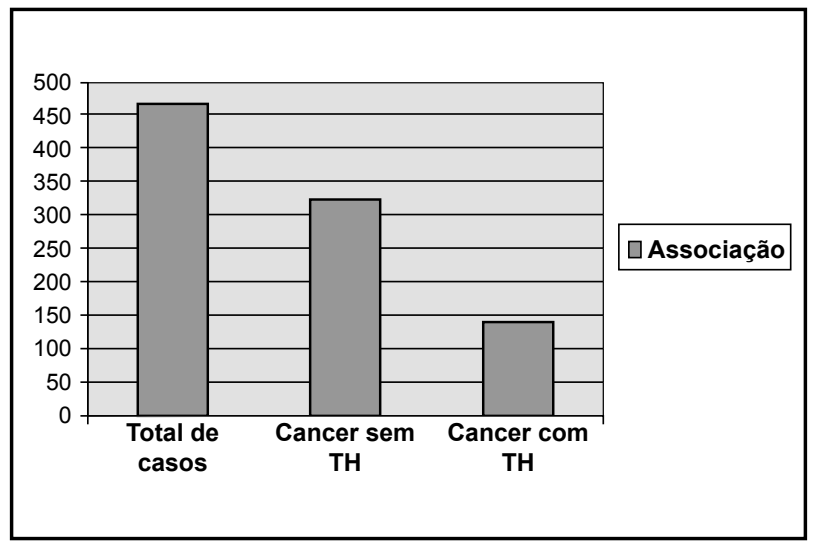

Figura - Frequência de TH no carcinoma papilífero TH: tireoidite de Hashimoto.

Dos dados demográficos presentes nos 466 prontuários de pacientes com carcinoma papilífero da tireoide, $392(84,1 \%)$ eram do sexo feminino e $74(15,9 \%)$, do sexo masculino. Dos casos sem a coexistência do carcinoma papilífero da tireoide e $\mathrm{TH}$, foram encontrados $267(68,1 \%)$ do sexo feminino e $59(79,7 \%)$ do sexo masculino, já nos casos com coexistência foram encontrados $125(31,8 \%)$ do sexo feminino e $15(20,2 \%)$, do sexo masculino (Tabela 1), mostrando que houve significância estatística $(p=0,046)$ por meio do teste do qui-quadrado da variável sexo no carcinoma papilífero com e sem presença de TH. (Tabela 2).

A média de idade dos 466 casos de carcinoma papilífero foi de 41,3 anos. Nos casos com a coexistência, a média foi de 44,8 anos e, nos casos sem a coexistência, de 40,7. A idade mínima foi de 18 anos e a máxima, de 77.
Distribuição dos casos de carcinoma

Tabela 1 papilífero com relação ao sexo

Carcinoma papilífero da tireoide

Sem TH Com TH

(\%) $\quad$ (\%) Total

$\begin{array}{lccccc}\begin{array}{l}\text { Sexo } \\ \text { masculino }\end{array} & 59 & 79,7 & 15 & 20,2 & 74 \\ \begin{array}{l}\text { Sexo } \\ \text { feminino }\end{array} & 267 & 68,1 & 125 & 31,8 & 392 \\ \text { Total } & 326 & - & 140 & - & 466\end{array}$

TH: tireoidite de Hashimoto.

\section{Distribuição dos casos das} variantes do carcinoma papilífero

Tabela 2 com e sem TH

\begin{tabular}{lccccc}
\hline & \multicolumn{3}{c}{ Carcinoma papilífero da tireoide } \\
& \multicolumn{2}{c}{ Sem TH } & \multicolumn{3}{c}{ Com TH } \\
Variantes & & $(\%)$ & \multicolumn{4}{c}{$(\%)$} & Total \\
Microcarcinoma & 141 & 43,2 & 70 & 50 & 211 \\
Clássica & 134 & 41,1 & 52 & 37,1 & 186 \\
Folicular & 49 & 15 & 18 & 12,8 & 67 \\
Sólida & 2 & 0,5 & 0 & 0 & 2 \\
Total & 326 & - & 140 & - & 466
\end{tabular}

TH: tireoidite de Hashimoto.

As variantes histológicas observadas nos 466 casos de carcinoma papilífero foram 211 (45,3\%), com variante microcarcinoma, $186(39,9 \%)$ apresentavam variante clássica, $67(14,4 \%)$, variante folicular e duas $(0,4 \%)$, variante sólida. Não foram evidenciados casos de variante de células altas nem de células claras.

Associando-se as variantes do carcinoma papilífero com a TH, foi constatado que dos 211 casos da variante microcarcinoma $141(43,2 \%)$ não apresentavam a coexistência, e em 70 (50\%) havia coexistência. Entre variante clássica e TH, $134(41,1 \%)$ apresentavam coexistência e em $52(37,1 \%)$ não havia coexistência. Nos casos com a variante folicular, 49 (15\%) possuíam a coexistência e 18 $(12,8 \%)$ não a possuíam. Na variante sólida, nos únicos dois casos $(0,5 \%)$ não havia coexistência da TH. Não houve significância estatística $(p=0,47)$ por meio do teste do qui-quadrado das variantes histológicas do carcinoma papilífero com e sem TH. 


\section{Discussão}

Desde a década de 1980, já existem estudos nos quais se demonstrava a coexistência entre TH e carcinoma papilífero da tireoide ${ }^{(25)}$. O presente estudo evidenciou uma coexistência de 30\%, (Figura), resultado que ratifica estudos anteriormente relatados como Cipolla ${ }^{(4)} \mathrm{e}$ Roberti( ${ }^{(4,22)}$. Isso sugere uma relação não apenas casual, mas possivelmente que a presença de processo inflamatório crônico com ativação de genes relacionados à multiplicação celular poderia ser mais um fator para a transformação maligna. Não há dados confirmatórios de sua fisiopatologia, no entanto, o que se tem investigado é o rearranjo cromossômico do RET/PTC, (fusão do RET-tirosina quinase na região 5 -terminal de genes heterogêneos), marcador útil para ativação oncogênica das células foliculares tireoidianas, também encontrado no tecido da tireoide dos pacientes com TH em $68 \%$ das vezes, relatado no estudo Rhoden $(2006)^{(5,19,22,23)}$. O RET/ PTC leva à expressão de RP3 no tecido da tireoide, fornecendo antígenos e estimulando a quimiotaxia para que monócitos e linfócitos infiltrem o tecido, evocando forte resposta imunológica. Além disso, as células da tireoide que expressam RP3 secretam um número grande de citocinas inflamatórias e induzem a transformação com contorno irregular do núcleo da célula, como também do citoesqueleto ${ }^{(5,9,23,25)}$.

Foi evidenciada predominância do sexo feminino, tanto no grupo de casos de carcinoma papilífero com tireoidite como no grupo de casos de carcinoma papilífero sem tireoidite; comparando-se os grupos, há um maior número de casos do sexo feminino com TH com relação ao grupo sem TH $(p=0,046)$. Do ponto de vista clínico, a frequência maior em mulheres pode ser explicada por uma série de fatores, entre eles doenças autoimunes (incluída aqui aTH), flutuações hormonais e gravidez, contribuindo possivelmente para esse aumento de casos de carcinoma na coexistência ${ }^{(18)}$. Sabe-se que, durante a gravidez, pode ocorrer acúmulo de células fetais na tireoide materna, que podem estar envolvidas no desencadear do processo autoimune ${ }^{(18)}$. Esses dados reforçam a ideia de uma relação causal entre doença autoimune, doença inflamatória e desenvolvimento de neoplasia maligna epitelial; no entanto, essa hipótese não pode ainda ser confirmada, pois não existem dados suficientes para elucidar se há ou não fatores genéticos, ambientais, hormonais ou inflamatórios ainda não descritos.

Com relação à distribuição dos casos das variantes do carcinoma papilífero com e sem $\mathrm{TH}$, foi observada distribuição equitativa de casos entre as três principais variantes mais comuns, sendo que não houve significância estatística $(p=0,47)$ para os grupos com e sem a coexistência, diferentemente dos dados da literatura, os quais evidenciaram maior número de casos de variante folicular no grupo das $\mathrm{TH}^{(5,12,14)}$. Inferiu-se que essa variante possui alterações genéticas mais frequentes do RET/PTC, com relação às variantes papilífera e micropapilífera, e que, desse modo, a TH seria causa dependente dessa alteração( ${ }^{(8,17)}$. Já no estudo de Basolo ${ }^{(1)}$, a alteração do RET/PTC foi encontrada em qualquer variante do carcinoma papilífero da tireoide, sem diferenças entre si. No entanto, foi observado que as variantes com essa alteração possuem um prognóstico mais agressivo, com maior número de recorrência após tratamento ${ }^{(1,13)}$.

Desse modo, ainda não está claro se a coexistência da TH denota pior prognóstico da neoplasia tireoidiana, e estudos posteriores devem ser realizados visando determinar a evolução da doença, que a princípio poderia ter sido desencadeada pela inflamação crônica da tireoide ${ }^{(4,21)}$.

\section{Conclusão}

A série de casos apresentada mostra frequência de $30 \%$ de $\mathrm{TH}$ nos casos de carcinoma papilífero da tireoide, sugerindo uma associação, não apenas casual, que levanta a possibilidade de uma relação de causa e efeito entre tireoidite e desenvolvimento do carcinoma. Além disso, o maior número de casos de mulheres no grupo com Hashimoto sugere que há uma escala de evolução, na qual inicialmente ocorrem alterações autoimunes, com posterior transformação neoplásica das células tireoidianas. No entanto, ainda são necessários mais estudos, seccionais ou de coorte, comprobatórios dessa associação e também da relação com alguma das variantes histopatológicas. 


\section{Referências}

1. BASOLO, F. et al. Potent mitogenicity of the RET/PTC3 oncogene correlates with its prevalence in tall-cell variant of papillary thyroid carcinoma. Am J Pathol, v. 160, n. 1, p. 247-54, 2002.

2. BENVENGA, S. Update on thyroid cancer. Horm Metab Res, v. 40, n. 5, p. 323-8, 2008.

3. CAHILL, S. et al. Effect of ret/PTC 1 rearrangement on transcription and post-transcriptional regulation in a papillary thyroid carcinoma model. Mol Cancer, v. 11, n. 5, p. 70, 2006.

4. CIPOLLA, C. et al. Hashimoto thyroiditis coexistent with papillary thyroid carcinoma. Am Surg, v. 71, p. 874-8, 2005.

5. COTRAN, R. S.; KUMAR, V.; ABBAS, F. O sistema endócrino, In: Robbins, SL, Contran, R.S, Kumar V, Abbas F. Patologia: bases patológicas das doenças. 7. ed. Rio de Janeiro: Guanabara Koogan, 2005. p. 1011-47.

6. Del RP, et al. The association between papillary carcinoma and chronic lymphocytic thyroiditis: does it modify the prognosis of cancer? Minerva Endocrinol, v. 33, n. 1, p. 1, 2008.

7. DELELLIS, R. Pathology and genetics of thyroid carcinoma. J Surg Oncol, v. 94, n. 8, p. 662-9, 2006.

8. FINN, S. P. et al. Expression microarray analysis of papillary thyroid carcinoma and benign thyroid tissue: emphasis on the follicular variant and potential markers of malignancy. Virchows Arch, v. 450, n. 3, p. 249-60, 2007.

9. FUSCO, A.; SANTORO, M. 20 years of RET/PTC in thyroid cancer: clinico-pathological correlations. Arq Bras Endocrinol Metabol, v. 51, n. 5, p. 731-5, 2007.

10. HARII, N. et al. Thyrocytes express a functional toll-like receptor 3: overexpression can be induced by viral infection and reversed by phenylmethimazole and is associated with Hashimotos autoimmune thyroiditis. Mol Endocrinol, v. 19, p. 1231-50, 2005.

11. $\mathrm{HE}, \mathrm{H}$. et al. The role of microRNA genes in papillary thyroid carcinoma. Proc Natl Acad Sci U S A, v. 27, p. 19075-80, 2005.

12. HUANG, W.T. et al. Encapsulated columnar-cell carcinoma of the thyroid: a case report. Kaohsiung J Med Sci, v. 21, n. 5, p. 241-4, 2005.

13. JUKKOLA, A. et al. Prognostic factors in differentiated thyroid carcinomas and their implications for current staging classifications. Endocr Relat Cancer, v. 11, n. 3, p. 571-9, 2004.

14. JUNG, T. et al. Clinical features and prognostic factors for survival in patients with poorly differentiated thyroid carcinoma and comparison to the patients with the aggressive variants of papillary thyroid carcinoma. Endocr J, v. 54, n. 2, p. 265-74, 2007.

15. KAKUDO, K. et al. Papillary carcinoma of the thyroid in Japan: subclassification of common type and identification of low risk group. Journal of Clinical Pathology, v. 57, p. 1041-6, 2004.

16. MACIEL, R.; KIMURA, E.; CERUTTI, M. Patogênese dos tumores diferenciados da tiróide (papilífero e folicular). Arquivos Brasileiros de Endocrinologia e Metabologia, v. 49, p. 691-700, 2005.

17. MAXWELL, E. L.; PALME, C. E.; FREEMAN, J. Hürthle cell tumors: applying molecular markers to define a new management algorithm. Arch Otolaryngol Head Neck Surg, v. 132, n. 1, p. 54-8, 2006.

18. MIGUEL, M. Tireoidites Autoimunes. Acta Med Port, v. 19, p. 387-94, 2006.

19. NIKIFOROV, Y. E. RET/PTC Rearrangement-a link between Hashimotos thyroiditis and thyroid cancer... or not. $J$ Clin Endocrinol Metab, v. 91, n. 6, p. 2040-2, 2006.

20. PRODOSMO, A. et al. Analysis of human MDM4 variants in papillary thyroid carcinomas reveals new potential markers of cancer properties. J Mol Med, v. 86, n. 5, p. 585-96, 2008.

21. REPPLINGER, D. et al. Is Hashimotos thyroiditis a risk factor for papillary thyroid cancer? J Surg Res, v. 150, n. 1, p. 49-52, 2008.

22. ROBERTI, A. et al. Concomitance of Hashimotos thyroiditis and differentiated thyroid cancer. Rev Col Bras Cir, v. 33, n. 6, p. 345-9, 2006.

23. RHODEN, K. J. et al. RET/papillary thyroid cancer rearrangement in nonneoplastic thyrocytes: follicular cells of Hashimotos thyroiditis share low-level recombination events with a subset of papillary carcinoma. Clin Endocrinol Metab, v. 91, n. 6, p. 2414-23, 2006.

24. STABENOW, E. et al. Angiogenesis as an indicator of metastatic potential in papillary thyroid carcinoma. Clinics (São Paulo, Brazil), v. 60, p. 233-40, 2005.

25. STRAUSS, M.; LAURIAN, N.; ANTEBI, E. Coexistent carcinoma of the thyroid gland and Hashimotos thyroiditis. Surgery Gynecology \& Obstetics, v. 157, n. 3, p. 228-32, 1983.

26. TOMER, Y. et al. Common and unique susceptibility loci in Graves and Hashimoto diseases: results of wholegenome screening in a data set of 102 multiplex families. American Journal of Human Genetics, v. 73, p. 736-47, 2003.

27. UNGER, K. et al. Heterogeneity in the distribution of RET/ PTC rearrangements within individual post-Chernobyl papillary thyroid carcinomas. J Clin Endocrinol Metab, v. 89, n. 9, p. 4272-9, 2004.

28. WEI, W. Z. Concurrent induction of antitumor immunity and autoimmune thyroiditis in CD4+ CD25+ regulatory $\mathrm{T}$ cell-depleted mice. Cancer Res, v. 65, p. 8471-8, 2005. 